\section{Newly launched interproximal cleaning device}

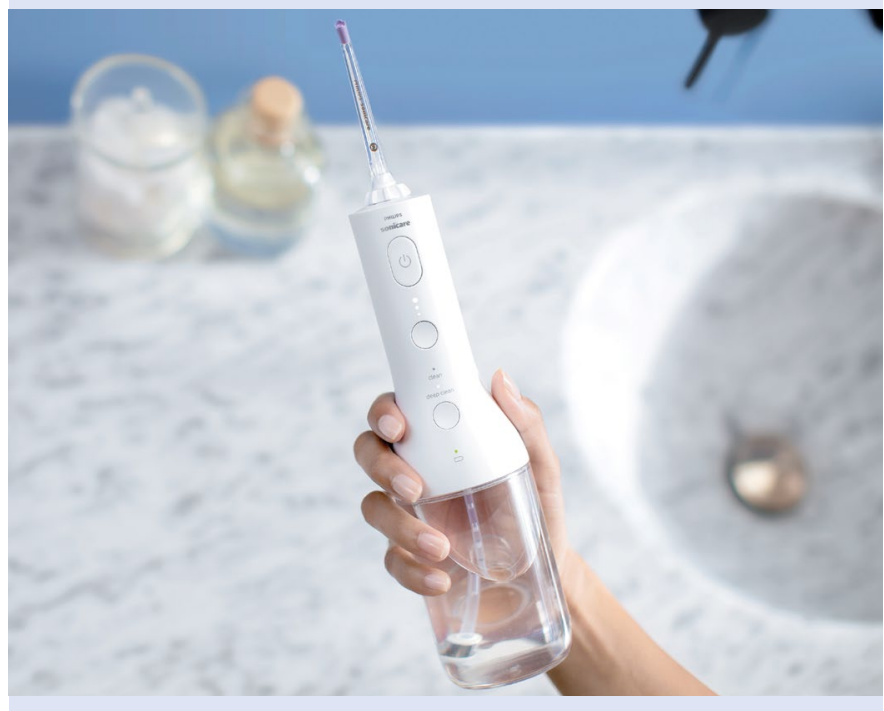

Philips has announced the launch of a new, powered, cordless Power Flosser, which is designed to help patients achieve an effective, consistent and convenient flossing routine and so improve their gum health in just two weeks. Launching in March, the new cordless Power Flosser builds on the company's air and micro-droplet device know-how, with the introduction of patented 'burst' function techology removing $99.9 \%$ of plaque in treated areas, to achieve an effortlessly thorough and superior clean.

Meanwhile, its unique cross-shaped nozzle creates four wide streams of water, ensuring it cleans eight times more area than a competitor irrigation appliance with a classic single-stream configuration. This ensures that it is less technique sensitive and can clean the whole mouth in 60-90 seconds.

It is this patented ' $\mathrm{x}$-shaped' QuadStream functionality which achieves maximum cleaning coverage by producing a plaquesheering cleaning action and combines it with a centre jet for a superior clean, interproximally and along the gumline. The designers considered its ergonomics and created an extra large water resevoir, meaning that the recommended 60-second clean can be carried out without refilling the device.

The cordless Power Flosser has 18 different water stream options including three intensities, two modes and two nozzle types, which allows for hyper-personalisation. In addition, newly developed pulse wave technology guides users from tooth to tooth, ensuring patients who struggle with regular flossing won't miss a spot and can clean the whole mouth effectively, as brief pauses in the cleaning pulsations prompt them to glide the nozzle to the next interproximal space. Meanwhile, the $360^{\circ}$ nozzle rotation ensures frustrated flossers are able to access hard-to-reach areas which have been off limits to those with manual dexterity issues. The nozzles are recyclable with TerraCycle through the Philips dental care recycling programme.

The Sonicare Cordless Power Flosser will be available for sale in dental practices from March 2022. For more information, visit: www.philips.co.uk/dentalprofessional.

\section{The latest innovation in teeth whitening}

Leading global smile care brand, Hismile has introduced a new tooth whitening treatment endorsed by the world's top dentists, setting a new standard for professional smile care.

Continuing to cement their reputation as the leader in the category with the launch of PAP+'s in-clinic treatment, Hismile have addressed the usual concerns around

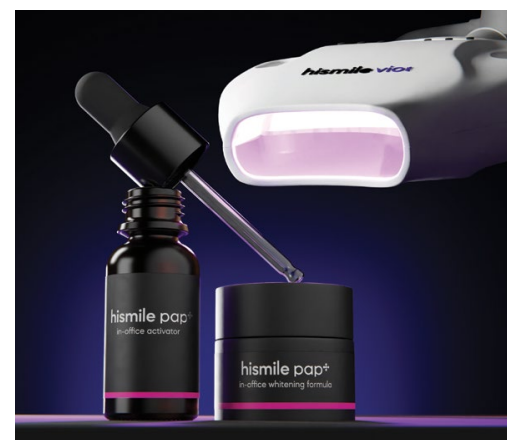
teeth whitening, offering a product which doesn't cause sensitivity or tooth and gum damage.

$\mathrm{PAP}+$ is a whitening formulation created by Hismile that includes the active ingredients phthalimidoperoxycaproic acid (PAP), hydroxyapatite (nano) and potassium citrate. The PAP+ formulation offers a whitening treatment that mitigates the typically expected adverse effects from tooth bleaching. With the active ingredient PAP, this method produces accelerated whitening results without compromising the tooth's mineral or protein structure. PAP is not an irritant to oral soft tissues, which means there is no requirement for a gingival barrier to protect the soft tissues. The inclusion of a nano-particle apatite (nano-hydroxyapatite) and potassium citrate offer certain additional benefits to the teeth during the whitening process, such as improved enamel structure and the reduction of pre-existing tooth sensitivity.

The PAP+ treatment is unique as it doesn't use peroxide, the traditional ingredient used to whiten teeth. Peroxide is known to demineralise teeth and leave them weak, exposed and sensitive. Instead, Hismile has used PAP, this magic ingredient for whitening, which works in tandem with their new Vio405 ultraviolet light technology, to not only leave your smile brighter and healthier but to add the guarantee of no pain or sensitivity. www.hismileteeth.com.

\section{Minimally-invasive endo}

The One RECI reciprocating file has flexibility and controlled memory, thanks to C.Wire heat treatment patented by MicroMega.

Available from COLTENE, the One RECI is safe, more resistant to cyclical fatigue, has unparalleled cutting efficiency and will respect complex root canal anatomy in all your endo cases.

This means you can practise the kind of conservative, minimally-invasive treatment that all dentists favour.

Explore opposite directions thanks to the file's reciprocating motion. Available in five sizes, root canals can be delivered comfortably, quickly and stress-free.

Always an innovator, COLTENE will upgrade your dentistry their customer service is exceptional too, so get in touch today.

For more on COLTENE, visit www.coltene.com, email info. uk@coltene.com or call 08002545115. 